\title{
Advanced two-step transcriptional amplification as a novel method for cancer-specific gene expression and imaging
}

\author{
MASAMI WATANABE ${ }^{1-3}$, HIDEO UEKI $^{2}$, KAZUHIKO OCHIAI $^{3}$, PENG HUANG $^{1}$, YASUYUKI KOBAYASHI ${ }^{2}$, \\ YASUTOMO NASU $^{2}$, KATSUMI SASAKI ${ }^{2}$, HARUKI KAKU ${ }^{1-3}$, YUJI KASHIWAKURA ${ }^{3}$ and HIROMI KUMON ${ }^{1-3}$ \\ ${ }^{1}$ Center for Gene and Cell Therapy, ${ }^{2}$ Department of Urology, ${ }^{3}$ Innovation Center Okayama \\ for Nanobio-Targeted Therapy, Graduate School of Medicine, Dentistry and Pharmaceutical \\ Sciences, Okayama University, 2-5-1 Shikata-cho, Okayama 700-8558, Japan
}

Received March 24, 2011; Accepted April 28, 2011

DOI: $10.3892 /$ or.2011.1371

\begin{abstract}
The two-step transcriptional amplification (TSTA) system was previously reported to enhance the tissue-specific gene expression driven by weak promoters, but the enhancement of the gene expression is limited to use in in vitro and in vivo experimental situations. To achieve robust tissuespecific gene expression using the TSTA system, we developed an advanced TSTA system which includes polyglutamines and rat glucocorticoid receptor sequences between the GAL4 and VP16 sequences in the region of the first step of transcription. We evaluated the advanced TSTA system as a method to enhance the human telomerase reverse transcriptase (hTERT) promoter-driving cancer-specific transcription in various cancer cell lines. As a result, the advanced TSTA enhanced cancer-specific luciferase gene expression in all of the examined cancer cell lines, when compared with both the one-step and conventional TSTA systems (an $\sim 6$ - and $\sim 17$-fold enhancement, respectively). Notably, the enhancement of the hTERT driven expression by the conventional TSTA system was modest and even inferior to the one-step system in several cancer cell lines. We then constructed a luciferase gene encoding the adenoassociated virus vector in which the hTERT promoter-mediated expression was driven by the advanced TSTA or control systems. In an orthotopic liver tumor model, mice were treated with the vector via tail vein injection. An optical imaging device was used to visualize the in vivo luciferase expression in the orthotopic tumor. The advanced TSTA system significantly enhanced the luciferase expression compared with the one-step

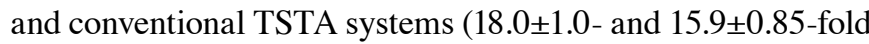
gain, respectively). Therefore, the advanced TSTA system significantly improves hTERT-dependent cancer-specific gene expression both in vitro and in vivo when compared with the
\end{abstract}

Correspondence to: Dr Masami Watanabe, Center for Gene and Cell Therapy, Okayama University, 2-5-1 Shikata-cho, Okayama 700-8558, Japan

E-mail: mwcorrespondence@gmail.com

Key words: cancer, two-step transcriptional amplification, human telomerase reverse transcriptase, imaging, adeno-associated virus previous systems. Since the advanced TSTA method can also be applied to other site-specific gene expression systems using tissue-specific promoters, this approach is expected to become a valuable tool enabling in vivo site-specific targeting in the field of gene therapy and molecular imaging.

\section{Introduction}

In gene therapeutic applications, targeted approaches to enhance safety and specificity are highly desirable. Transcriptional targeting by using tissue-specific promoters to limit the expression of potential cytotoxic transgenes to the tissue of interest has been frequently used. The human telomerase reverse transcriptase (hTERT) promoter has been well characterized and determined to be available for cancer tissue-specific gene expression in a broad range of malignant tissue types (1-3). In previous studies, we have demonstrated that treatment with an hTERT-driven replication competent adenovirus, OBP-301, led to significant tumor regression in mouse models of prostate and renal cancer $(4,5)$. However, the in vivo use of tissuespecific promoters, including hTERT, is often limited because the specific promoters can not lead to sufficient levels of therapeutic or reporter gene expression. Therefore, enhancement of the expression seems to be necessary for the in vivo gene therapy and imaging, and this may benefit from tissue-specific amplification of the gene expression.

In order to utilize a relatively weak promoter in in vitro and in vivo gene expression studies, it is essential to enhance the transcriptional activity of the promoter. There are several potential methods which can be used to increase gene expression levels (6-10). One of the most attractive methods is the transcriptional amplification approach referred to as the two-step transcriptional amplification (TSTA) (10). This conventional TSTA approach with a GAL4-VP16 fusion protein can potentially be used to augment the tissue-specific transcriptional activity of cellular promoters (10). However, in our experience, the conventional TSTA system adversely suppresses the gene expression in many cancer cell lines, and therefore, its utility in a broad range of cancer types is limited.

For the purpose of overcoming the weak points of the hTERT promoter and of the conventional TSTA system, we have been improving the gene expression system by modifying 
the construct sequence. In a series of modifications of the hTERT promoter-dependent gene expression cassette with the TSTA system, we adopted the insertion of polyglutamines and rat glucocorticoid receptor (GR) sequences within the GAL4VP16 fusion sequence, which were previously reported to enhance gene transcription (11). By utilizing the conventional TSTA system (10) and the sequence of polyglutamines and rat GR (11), we developed a new hTERT promoter-driving advanced TSTA system that retains the strong transactivating properties of the GAL4-VP16 fusion protein. We, herein, report our evaluation and validation of this novel system for the enhancement of cancer-specific gene expression in various cancer cell lines. Furthermore, we demonstrate the utility of the cancer-specific approach for in vivo imaging of luciferase expression in an orthotopic liver tumor model.

\section{Materials and methods}

Cell culture. Normal human prostate epithelial cells (PrEC) were purchased from Lonza (Basel, Switzerland) and cultivated using medium recommended by the supplier. Normal human fibroblasts (OUMS24) were established by the department of cell biology at our university (12). All human cancer cell lines and the HaCaT cell line were provided by the American Type Culture Collection (Rockville, MD) (Hep3B, HepG2, liver cancer; HeLa, cervical cancer; LNCaP, PC3, DU145, prostate cancer; NCCIT, testicular cancer; MDA231, SKBR3, MCF7, breast cancer; A549, lung cancer; H28, 211H, H2452, H2052, malignant mesothelioma; KPK1, renal cancer; J82, T24, bladder cancer; A431, epidermoid carcinoma; DLD1, colon cancer). The $\mathrm{HaCaT}$ cell line is a spontaneously transformed human epithelial cell line from adult skin (13). This cell line is used here as non-cancer cell line because it retains some differentiation characteristics of human skin cells. Dulbecco's modified Eagle's medium (DMEM; Invitrogen, Carlsbad, CA), RPMI-1640 and HAM'S F-12 K medium (Nissui, Tokyo, Japan) were used for the culture of human cancerous and non-cancerous cell lines. The cell lines were grown in the medium supplemented with $10 \%$ fetal bovine serum (FBS; Biowest, Nuaille, France), as previously described (14).

Advanced TSTA experimental strategy. The VP16 immediate early transactivator contains a highly potent activation domain that, when fused to the GAL4 binding domain, elicits a robust response on a GAL4-responsive promoter (an adenovirus minimal promoter) bearing multiple tandem copies of the 17 bp of the GAL4 binding site (10). This system is referred to as the TSTA in the present study. For the purpose of improving the conventional TSTA system, we constructed an advanced TSTA system. In the advanced system, we inserted the polyglutamines and rat glucocorticoid receptor (GR) sequences within the GAL4-VP16 fusion sequence, which were previously reported to enhance gene transcription (11). By combining the conventional TSTA system and the sequence of polyglutamines and rat GR, we established a new hTERT promoter-dependent advanced TSTA system with strong transactivating properties due to the GAL4-VP16 fusion protein and GAL4 binding sites.

Construction of plasmid vectors. For the construction of the one-step plasmid, the hTERT promoter region from -377 to
+77 (1) was synthesized and inserted into the pGL3-basic vector (Promega) at the restriction site of $M l u \mathrm{I}-\mathrm{BglII}$. To construct the conventional two-step (TSTA) plasmid, the GAL4-VP16 fusion sequence (9) and 5X repeated GAL4 binding sites with an adenoviral minimal promoter (10) were inserted into the one-step plasmid at the restriction sites of BglII-HindIII and HindIII-NcoI, respectively. The GAL4-VP16 fusion protein consists of the GAL4 binding domain amino acids 1-147 and the N-terminal portion of the VP16 activation domain amino acids 413-456 (8,9). For the advanced two-step (TSTA) plasmid, the sequence of polyglutamines and rat GR of the following amino acids was inserted between the GAL4 and VP16 sequences of the conventional two-step plasmid: [MGLY (37Q) MGETETKVMGNDLGYPQQGQLGLSSGETDFRL LEESIANLNRS].

Cell transfections and luciferase assays. The cells were plated in 24-well plates in culture medium containing charcoalstripped FBS. After $24 \mathrm{~h}$, transient transfections of the luciferase-encoding plasmid with the one-step, conventional TSTA or advanced TSTA system were performed by using the Lipofectamine transfection reagent (Invitrogen). The effector plasmid was co-transfected with the reporter plasmid derived from the dual-reporter luciferase assay kit (Promega, Madison, WI), and each transfection mix consisted of $0.5 \mu \mathrm{g}$ of the effector and reporter plasmids or the reporter plasmid alone. After $48 \mathrm{~h}$ of incubation, the cells were harvested and assayed for luciferase activity by using the luciferase assay kit and a luminescence microplate reader, according to the manufacturer's instructions. As a positive control, cells were also transfected with a CMV promoter-luciferase plasmid.

Telomerase activity assays. To analyze the telomerase activity, the Telo TAGGG Telomerase PCR ELISAPLUS kit (Roche, Basel, Switzerland) was employed according to the manufacturer's instructions. The assay uses the internal activity of telomerase, amplifying the product by PCR and detecting it with an enzyme linked immunosorbent assay. Typically, $2 \times 10^{5}$ cells per sample were harvested, and the pelleted cells were resuspended in $200 \mu \mathrm{l}$ of the lysis reagent. After centrifugation, the supernatants of the lysates were collected and $3 \mu \mathrm{l}$ of cell extract (corresponding to $3 \times 10^{3}$ cell equivalents) were then used for the following procedures.

Construction and production of adeno-associated virus $(A A V)$ vectors. The cDNA encoding luciferase was obtained from the pGL3-basic vector by digestion with the restriction enzymes. The construction and production of AAV vectors was performed as previously described (15-17). Briefly, for the rAAV vector plasmid (hTERT promoter/luciferase with the one-step, conventional TSTA and advanced TSTA system) construction, the AAV vector plasmid (SF) derivatives of psub-201 were employed. The rAAV virus vectors were propagated in an adenovirus-free system (Stratagene, La Jolla, CA, USA). All virus preparations were purified by two successive bandings on $\mathrm{CsCl}$ gradients to ensure purity. The titer of the respective AAV virus vectors was determined by DNA-PCR.

In vivo detection of luciferase expression in the mouse orthotopic liver tumor model. The mouse model with orthotopic 


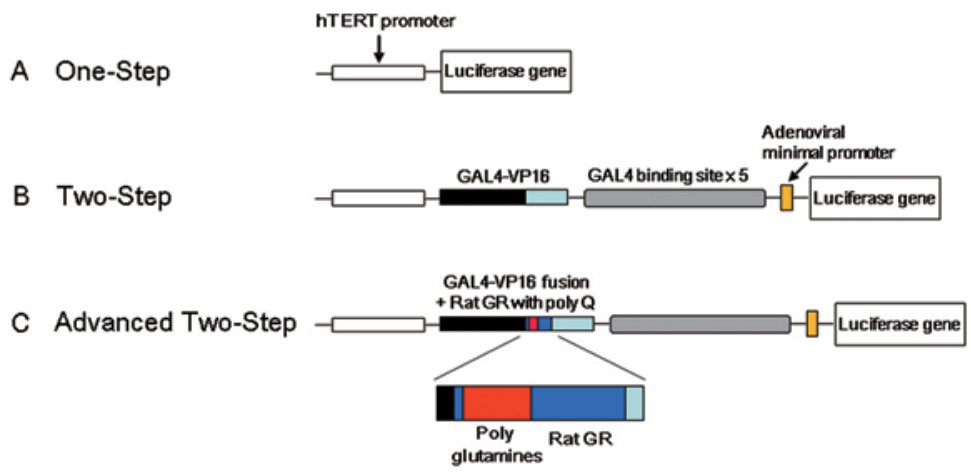

Figure 1. A schematic diagram of the advanced TSTA system. In the conventional TSTA system for cancer-specific gene transcription (B), the first step includes the expression system of the GAL4-VP16 fusion protein driven by the hTERT promoter. In the second step, GAL4-VP16, in turn, drives the target gene expression under the control of GAL4 response elements (GAL4 binding sites) upstream of the minimal promoter. The advanced TSTA system (C) additionally includes polyglutamines and rat glucocorticoid receptor sequences between the GAL4 and VP16 sequences in the region of the first step of transcription. The insert of the polyglutamines and rat glucocorticoid receptor protein in the GAL4-VP16 fusion protein can lead to amplification of the luciferase gene expression.

liver tumors was established in male nude mice. Hep3B liver cancer cells $\left(5 \times 10^{6}\right.$ cells $/ 100 \mu 1$ PBS equally mixed with Matrigel (BD Biosciences, Bedford, MA) were orthotopically inoculated into the subcapsular space of the left lobe of the liver on experimental day 0 (Fig. 5A). Five days after the cell injection, $1 \times 10^{10}$ of the luciferase-encoding AAVs (in $100 \mu \mathrm{l}$ PBS) were intravenously injected via a tail vein. On day 30 after the cell inoculation, tumor imaging was performed by detecting the luciferase expression in the Hep3B tumors using an optical imaging device. Briefly, the animals were anesthetized with isoflurane before and during imaging and then were injected intraperitoneally with luciferin (a substrate for luciferase) at $150 \mathrm{mg} / \mathrm{kg}$ in a volume of $100 \mu \mathrm{l}$. The animals were imaged at a peak time of 20-min post-luciferin injection via an IVIS-Spectrum instrument (Xenogen, Alameda, CA), using exposure times and sensitivity settings to avoid saturation. Image processing was done using the Living Image software program (Xenogen), by means of a region-of-interest analysis of the total photons per second for each tumor, with appropriate background subtraction.

For the imaging of the liver tumors with computed tomography (CT), images of the whole animal were obtained with a micro-CT scanner (GE Healthcare, Tokyo, Japan). Mice were fasted before the scan to minimize imaging artifacts due to minerals that are found in rodent chow. Prior to scanning, mice were intravenously administered the liver-specific contrast agent (Fenestra LC; Advanced Research Technologies, Inc., Quebec, Canada), according to the manufacturer's instructions.

Statistical analysis. The data are shown as the means \pm standard deviation. The unpaired Student's t-test was performed to analyze the statistical significance of differences between any 2 groups. A regression analysis was performed to examine the correlation of 2 parameters. Differences or correlations were considered to be significant when $\mathrm{p}<0.05$.

\section{Results}

The advanced TSTA system mediates hTERT-dependent selective luciferase expression in human cancer cell lines.
The advanced TSTA system was constructed by inserting the polyglutamines and rat glucocorticoid receptor sequences between the GAL4 and VP16 sequences of the region of the first step transcription (Fig. 1C). We first measured the telomerase activity using commercially-available kits. Relatively strong telomerase activity was observed in the cancer cell lines, but the level was almost undetectable in the OUMS24 and PrEC normal cells (Fig. 2A). To determine whether the advanced TSTA system-mediated amplification is restricted to cancer cells with higher telomerase activity, we performed a luciferase assay of the advanced TSTA plasmid in the various cell lines, including the two normal cells. The luciferase expression level was minimal in both normal cell lines, but as the telomerase activity increased in the cancer cells, more luciferase expression was observed. A significant correlation was demonstrated between the relative telomerase activity and the actual values of luciferase expression determined by the regression analysis (Fig. 2B). Since telomerase activity is strongly correlated to the hTERT expression level and hTERT promoter activity (1), the advanced TSTA system-mediated gene expression is expected to be regulated by the hTERT promoter and to therefore be highly cancer-specific.

The advanced TSTA system significantly enhances in vitro gene expression in comparison to both the one-step and conventional TSTA systems. We next evaluated the advanced TSTA system as a method to enhance the transcriptional activity of the hTERT promoter in various cancer cell lines. Using the luciferase assay, we demonstrated that there was a significant enhancement of gene expression using the advanced TSTA approach compared to that induced by the one-step and conventional TSTA systems (Fig. 3A). When compared to the one-step system, a 3- to 6-fold gain in luciferase expression was observed in the examined cells. Interestingly, our study disclosed that the conventional TSTA system suppressed the gene expression in several cell lines. To demonstrate the superiority of the advanced TSTA system compared to the conventional system, we compared the two systems. Significant enhancement of expression by the advanced TSTA was indicated by the 3 - to 17 -fold gain of luciferase expression in 
A

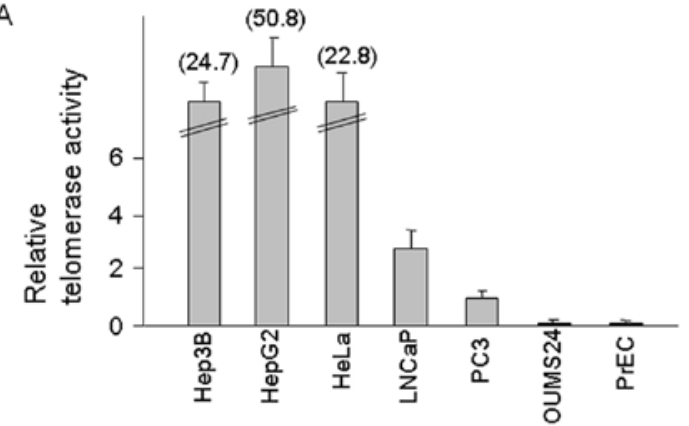

B

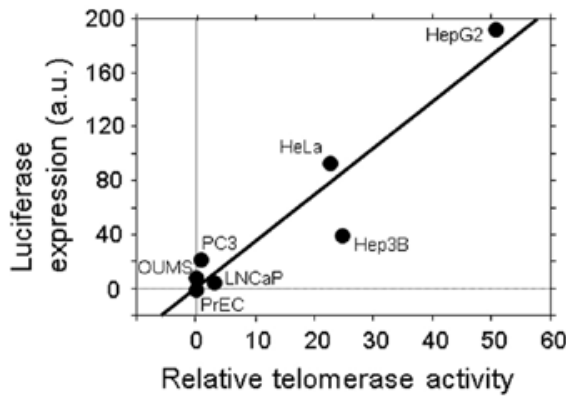

Figure 2. (A) Telomerase activity was determined in human cancer and normal cell lines. The telomerase activity of PC3 human prostate cancer cells was considered to be 1.0, and the relative activity of each cell line was calculated in comparison to that of PC3 cells. The data are from triplicate measurements. (B) The relationship between the relative telomerase activity and luciferase expression (arbitrary unit: a.u.) was examined by a regression analysis. The level of luciferase expression was determined by the luminescence of luciferin, a substrate for luciferase. The analysis showed a significant association between the parameters $(\mathrm{p}<0.05)$.

comparison to the expression due to the conventional system (Fig. 4). Thus, in all of the cancer cell lines examined here, the advanced TSTA system significantly improved hTERTdependent gene expression in comparison to both of the previous systems. These results demonstrate that there is a strong transcriptional amplification exerted by the GAL4VP16 fusion protein with polyglutamines and the rat GR in the hTERT-dependent cancer-specific gene expression.

The advanced TSTA system demonstrates hTERT-dependent gene expression fold-gain in comparison to the one-step system. Since the advanced TSTA system retained the ability to induce hTERT-dependent cancer cell-specific gene expression, we next examined whether the system could improve the one-step system in an hTERT-dependent manner. As was expected, there was a significant correlation between the telomerase activity of the cells and the fold gain in luciferase expression determined by the regression analysis (Fig. 3B). Therefore, as telomerase activity increases in the cancer cell lines, a higher fold gain of luciferase gene expression is observed compared to that of the one-step system. These results indicate that the advanced TSTA system is more advantageous in improving the one-step system in the cancer cell-situation with higher telomerase activity.

The advanced TSTA system achieves in vivo cancer-specific gene expression in an orthotopic model of liver cancer. To further examine the utility of the advanced TSTA system

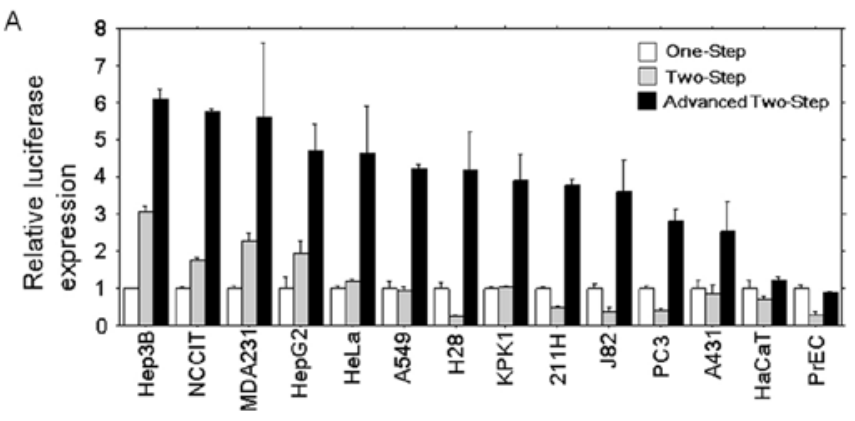

B

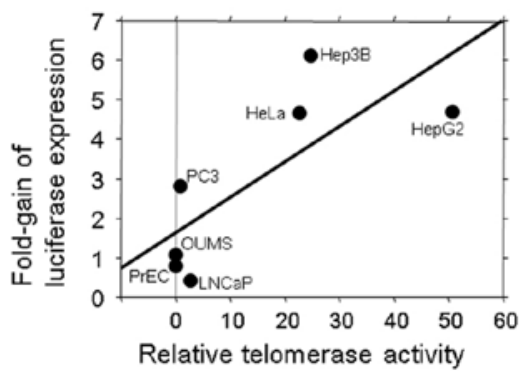

Figure 3. (A) The in vitro gene expression was compared between the onestep, conventional TSTA and advanced TSTA systems. The luciferase expression of the one-step system was considered as 1.0, and the relative luciferase expression ratios of the conventional and advanced TSTA systems are presented for each cell line in comparison with the one-step system. The data are from triplicate measurements. (B) The relationship between the relative telomerase activity and fold-gain of luciferase expression in the advanced TSTA was examined by a regression analysis. The y-axis value of A for the advanced TSTA in the cell lines is used as the fold-gain of the luciferase expression here. The analysis showed a significant association between the parameters $(\mathrm{p}<0.05)$.

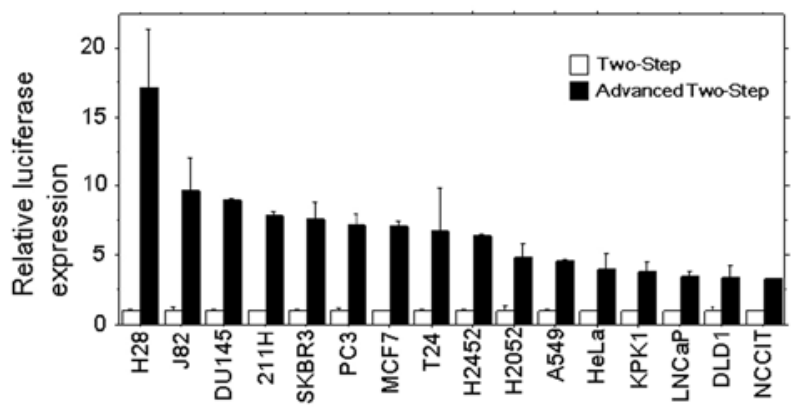

Figure 4 . The in vitro gene expression was compared between the conventional TSTA and advanced TSTA systems. The luciferase expression of the conventional TSTA system was considered to be 1.0, and the relative luciferase expression ratios of the advanced TSTA system are presented for each cell line in comparison with the conventional TSTA system. The data are from triplicate measurements.

in vivo, we constructed $\mathrm{AAV}$ vectors with one-step, conventional TSTA, and advanced TSTA systems, encoding the luciferase gene driven by the hTERT promoter. Hep3B liver cancer cells were injected into the livers of nude mice to produce an orthotopic tumor model. On day 30 after implantation of the Hep3B cancer cells, the orthotopic tumors derived from the cells were typically confirmed macroscopically and by computed tomography (Fig. 5B). In this liver cancer model, the mice were intravenously treated with the AAV-luciferase vectors on day 5 after the cell inoculation, and then the in vivo tumor growth was assessed. On day 30 after the cell inoculation, the 
A

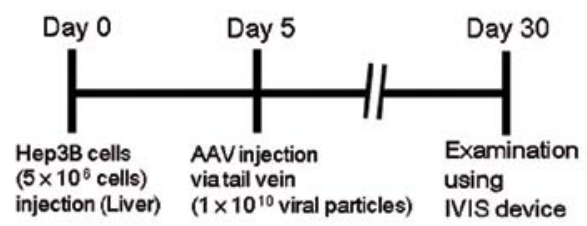

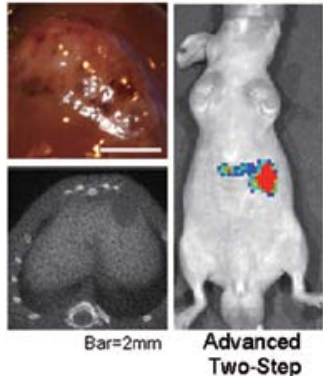

C
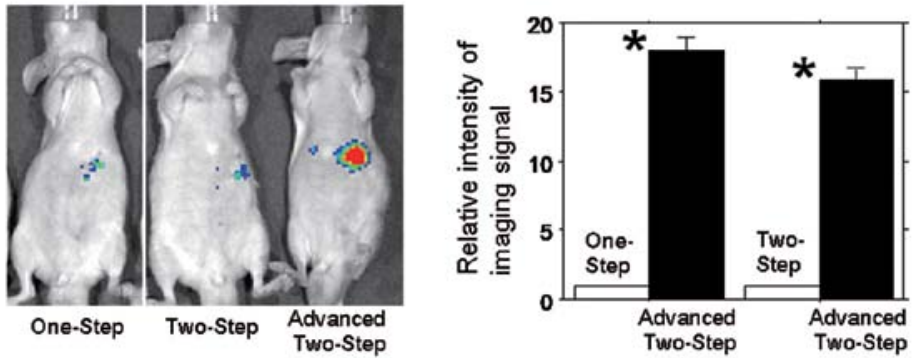

Figure 5. (A) The in vivo gene expression was compared between the one-step, conventional TSTA and advanced TSTA systems. The experiment was performed in the orthotopic liver cancer model, and the AAV vectors were used for luciferase gene expression. The treatment schedule is shown. (B) The macroscopic view of the Hep3B liver tumors on day 30 is shown in the left upper panel. The computed tomography (CT) imaging of the liver tumor is shown in the left lower panel. The luciferase expression by the AAV-advanced TSTA vector was analyzed by the IVIS device, and the representative image of the luminescence signal at the tumor site is shown in the right panel. (C) The representative images of the luminescence signal by the AAVs with the one-step, conventional and advanced TSTA systems are present in the left panel. In the right panel, the intensity of the luminescence signal in the IVIS device was compared between the indicated groups. The imaging intensity of the one-step or conventional TSTA system was considered as 1.0, and the relative imaging intensity of the advanced TSTA was presented. There was a significant fold gain in the luciferase expression using the advanced TSTA system ("p $<0.05$ ), when compared with the one-step or the conventional TSTA systems. The data are from the measurements of three different mice.

advanced TSTA system demonstrated a strong luciferase expression signal in the liver tumor with the IVIS instrument (Fig. 5B). On the other hand, the control mice treated with the one-step and conventional TSTA systems showed minimal levels of tumor luciferase signaling. The advanced TSTA system therefore, significantly enhanced luciferase expression when compared with the one-step and conventional TSTA systems (Fig. 5C) $(18.0 \pm 1.0$-fold and 15.9 \pm 0.85 -fold gain, respectively). These results demonstrate the utility of the advanced TSTA approach to achieve in vivo cancer-specific gene expression driven by the hTERT promoter.

\section{Discussion}

Therapeutic and imaging approaches with tissue-specific gene expression depend on robust levels of the products at both the mRNA and protein levels. Although the hTERT promoter is well-characterized, and used as a broad range cancer-specific promoter (2), the gene expression driven by the hTERT promoter is often weak due to its poor transcriptional activity. We have been developing methods to enhance gene expression driven by various promoters, and herein described a novel strategy to overcome the weak points of the hTERT promoter and to enhance the cancer-specific gene expression and imaging. We developed the advanced two-step transcriptional amplification (TSTA) system by inserting polyglutamines and rat GR sequences within the GAL4-VP16 fusion sequence of the conventional TSTA system. The advanced system could strongly activate the hTERT promoter-driven reporter gene expression in the expected cancer-specific manner based on the telomerase activity.
In the present study, we observed that the TSTA systemmediated amplification of luciferase expression was $\sim 6$-fold higher than the one-step system in the cancer cells. We have also demonstrated that the enhanced luciferase expression induced by the advanced TSTA system significantly correlates to the telomerase activity, and is cancer-specific. Significant enhancement was also obtained ( 17-fold) when we compared the current advanced TSTA system with the conventional TSTA system. Notably, the enhancement of the hTERT-driven expression by the conventional TSTA system was found to be modest, and even inferior to the one-step system, in several cancer cell lines. As a result, the hTERT-driven advanced TSTA enabled the enhancement of cancer-specific gene expression in all of the examined cancer cell lines compared with both the one-step and conventional TSTA systems.

Our present findings clearly indicate that the advanced system with the polyglutamines and rat GR insert functions under the hTERT promoter. Furthermore, as the telomerase activity in cancer cells is elevated, a greater fold-gain in luciferase gene expression is observed in the advanced TSTA system. These results demonstrate that the advanced TSTA system has more advantages in improving the one-step system in the cancer cells with higher hTERT activity. The most likely mechanism underlying this effect is that the polyglutamines and rat GR region significantly increase the levels of the GAL4-VP16 fusion protein, thereby increasing the ability to bind to GAL4 binding sites upstream of the second minimal promoter, resulting in higher levels of mRNA transcription and subsequent protein expression.

We further tested the in vivo utility of the advanced TSTA system in a tissue-specific amplification of the reporter gene 
expression. We constructed adeno-associated virus vectors containing the advanced TSTA component and the luciferase gene driven by the hTERT promoter. To expedite the process of in vivo evaluation, we established an orthotopic liver tumor model in nude mice. In the AAV-advanced TSTA group, the mice showed significantly greater imaging signals for luciferase expression in the liver tumors compared with the mice treated with the one-step and conventional TSTA. In fact, in the absence of the advanced TSTA system, the luciferase expression was close to background, and therefore, the tumor could not be efficiently imaged.

The AAV vector was injected intravenously to provide targeted gene expression in the liver tumor. The AAV vector has advantages for directing expression to the liver in terms of the in vivo distribution and transfection of the reporter gene (18), so this may have helped the targeted expression of the luciferase gene in the tumor site. Furthermore, tumor-specific imaging was accomplished by the enhanced luciferase expression with the hTERT promoter-driven advanced TSTA system. By replacing the luciferase gene with an anti-cancer therapeutic gene in this model, future studies can be used to demonstrate the availability of this AAV-advanced TSTA system for cancerspecific gene therapy.

Approaches for imaging reporter gene expression are very useful for visualizing the molecular dynamics in living animals. Imaging of cancer-specific reporter gene expression with radionuclides and with non-radionuclide techniques allows the monitoring of the tumor location, magnitude, and time variations in living animals and humans. It is reasonable that all of the imaging applications could benefit from repetitive, non-invasive monitoring of reporter gene expression. Methods to image reporter gene expression in vivo include positron emission tomography (PET) (19), single photon emission computed tomography (20), magnetic resonance imaging (21), bioluminescent optical imaging with luciferase (10), and fluorescence optical imaging with green fluorescent protein (2). The current advanced TSTA system may be useful to improve the imaging sensitivity of these approaches due to the enhancement of in vivo imaging signals.

Until now, the conventional TSTA system with the GAL4VP16 fusion protein has been used to achieve the tissue-specific gene expression with the various specific promoters $(22,23)$. In the present study, we developed and validated a novel TSTA system which robustly enhances cancer-specific gene expression in comparison to the conventional TSTA system. We believe that the advanced TSTA method with the hTERT promoter can be used for cancer-targeted gene therapy. Moreover, applications and extensions of the advanced TSTA approach can include other site-specific gene expression approaches by replacement of the hTERT promoter with other tissue-specific promoters. The advanced TSTA approach may represent a valuable new tool enabling in vivo site-specific targeting in the field of gene therapy and molecular imaging.

\section{Acknowledgements}

This study was supported by a grant from the Ministry of Education, Culture, Sports, Science and Technology's FY2006 'Creation of Innovation Centers for Advanced Interdisciplinary Research Areas' Scheme in Japan. We thank Dr M. Takaoka and Dr S.A. Li (Okayama University) for their valuable assistance.

\section{References}

1. Takakura M, Kyo S, Kanaya T, Hirano H, Takeda J, Yutsudo M and Inoue M: Cloning of human telomerase catalytic subunit (hTERT) gene promoter and identification of proximal core promoter sequences essential for transcriptional activation in immortalized and cancer cells. Cancer Res 59: 551-557, 1999.

2. Kishimoto H, Kojima T, Watanabe Y, Kagawa S, Fujiwara T, Uno F, Teraishi F, Kyo S, Mizuguchi H, Hashimoto Y, Urata Y, Tanaka $\mathrm{N}$ and Fujiwara $\mathrm{T}$ : In vivo imaging of lymph node metastasis with telomerase-specific replication-selective adenovirus. Nat Med 12: 1213-1219, 2006.

3. Maida Y, Kyo S, Sakaguchi J, Mizumoto Y, Hashimoto M, Mori N, Ikoma T, Nakamura M, Takakura M, Urata Y, Fujiwara T and Inoue M: Diagnostic potential and limitation of imaging cancer cells in cytological samples using telomerase-specific replicative adenovirus. Int J Oncol 34: 1549-1556, 2009.

4. Huang P, Watanabe M, Kaku H, Kashiwakura Y, Chen J, Saika T, Nasu Y, Fujiwara T, Urata Y and Kumon H: Direct and distant antitumor effects of a telomerase-selective oncolytic adenoviral agent, OBP-301, in a mouse prostate cancer model. Cancer Gene Ther 15: 315-322, 2008.

5. Huang P, Kaku H, Chen J, Kashiwakura Y, Saika T, Nasu Y, Urata Y, Fujiwara T, Watanabe M and Kumon H: Potent antitumor effects of combined therapy with a telomerase-specific, replicationcompetent adenovirus (OBP-301) and IL-2 in a mouse model of renal cell carcinoma. Cancer Gene Ther 17: 484-491, 2010.

6. Wu L, Matherly J, Smallwood A, Adams JY, Billick E, Belldegrun A and Carey M: Chimeric PSA enhancers exhibit augmented activity in prostate cancer gene therapy vectors. Gene Ther 8: 1416-1426, 2001.

7. Choi T, Huang M, Gorman C and Jaenisch R: A generic intron increases gene expression in transgenic mice. Mol Cell Biol 11: 3070-3074, 1991.

8. Emami KH and Carey M: A synergistic increase in potency of a multimerized VP16 transcriptional activation domain. EMBO J 11: 5005-5012, 1992.

9. Sadowski I, Ma J, Triezenberg S and Ptashne M: GAL4-VP16 is an unusually potent transcriptional activator. Nature 335: 563-564, 1988.

10. Iyer M, Wu L, Carey M, Wang Y, Smallwood A and Gambhir SS: Two-step transcriptional amplification as a method for imaging reporter gene expression using weak promoters. Proc Natl Acad Sci USA 98: 14595-14600, 2001.

11. Gerber HP, Seipel K, Georgiev O, Höfferer M, Hug M, Rusconi S and Schaffner W: Transcriptional activation modulated by homopolymeric glutamine and proline stretches. Science 263 : 808-811, 1994.

12. Zhang K, Watanabe M, Kashiwakura Y, Li SA, Edamura K, Huang P, Yamaguchi K, Nasu Y, Kobayashi Y, Sakaguchi M, Ochiai K, Yamada H, Takei K, Ueki H, Huh NH, Li M, Kaku H, $\mathrm{Na} \mathrm{Y}$ and Kumon H: Expression pattern of REIC/Dkk-3 in various cell types and the implications of the soluble form in prostatic acinar development. Int J Oncol 37: 1495-1501, 2010.

13. Boukamp P, Petrussevska RT, Breitkreutz D, Hornung J, Markham A and Fusenig NE: Normal keratinization in a spontaneously immortalized aneuploid human keratinocyte cell line. J Cell Biol 106: 761-771, 1988.

14. Watanabe M, Kashiwakura Y, Huang P, Ochiai K, Futami J, Li SA, Takaoka M, Nasu Y, Sakaguchi M, Huh NH and Kumon H: Immunological aspects of REIC/Dkk-3 in monocyte differentiation and tumor regression. Int J Oncol 34: 657-663, 2009.

15. Kashiwakura Y, Tamayose K, Iwabuchi K, Hirai Y, Shimada T, Matsumoto K, Nakamura T, Watanabe M, Oshimi K and Daida H: Hepatocyte growth factor receptor is a coreceptor for adeno-associated virus type 2 infection. J Virol 79: 609-614, 2005.

16. Watanabe M, Kashiwakura Y, Kusumi N, Tamayose K, Nasu Y, Nagai A, Shimada T, Daida H and Kumon H: Adeno-associated virus-mediated human IL-10 gene transfer suppresses the development of experimental autoimmune orchitis. Gene Ther 12: $1126-1132,2005$

17. Watanabe M, Nasu Y, Kashiwakura Y, Kusumi N, Tamayose K, Nagai A, Sasano T, Shimada T, Daida H and Kumon H: Adenoassociated virus 2-mediated intratumoral prostate cancer gene therapy: long-term maspin expression efficiently suppresses tumor growth. Hum Gene Ther 16: 699-710, 2005. 
18. Daly TM: AAV-mediated gene transfer to the liver. Methods Mol Biol 246: 195-199, 2004.

19. Gambhir SS, Barrio JR, Phelps ME, Iyer M, Namavari M, Satyamurthy N, Wu L, Green LA, Bauer E, MacLaren DC, Nguyen K, Berk AJ, Cherry SR and Herschman HR: Imaging adenoviral-directed reporter gene expression in living animals with positron emission tomography. Proc Natl Acad Sci USA 96 2333-2338, 1999

20. Tjuvajev JG, Finn R, Watanabe K, Joshi R, Oku T, Kennedy J, Beattie B, Koutcher J, Larson S and Blasberg RG: Non-invasive imaging of herpes virus thymidine kinase gene transfer and expression: a potential method for monitoring clinical gene therapy. Cancer Res 56: 4087-4095, 1996.
21. Louie AY, Hüber MM, Ahrens ET, Rothbächer U, Moats R, Jacobs RE, Fraser SE and Meade TJ: In vivo visualization of gene expression using magnetic resonance imaging. Nat Biotechnol 18: 321-325, 2000.

22. Köster RW and Fraser SE: Tracing transgene expression in living zebrafish embryos. Dev Biol 233: 329-346, 2001.

23. Iyer M, Salazar FB, Lewis X, Zhang L, Carey M, Wu L and Gambhir SS: Non-invasive imaging of enhanced prostate-specific gene expression using a two-step transcriptional amplificationbased lentivirus vector. Mol Ther 10: 545-552, 2004 